\title{
versants
}

\section{Introduction : la valeur du commun}

Annick ETTLIN

Université de Genève

\begin{abstract}
L'idée de littérature moderne se fonde sur une séparation entre les langues littéraire et courante : il est de fait significatif que le lieu commun, issu de la rhétorique, acquiert son sens péjoratif justement au seuil de la modernité, lorsque la littérature devient autonome. Pourtant, comme le savent déjà les écrivains des XIX et XXe siècles - Baudelaire et Flaubert en tête -, le lieu commun permet de diffuser des idées, de communiquer des contenus, de se réapproprier un rapport au langage qui n'est pas sans valeur esthétique. Il est surtout le signe d'une pensée collective et participe à la constitution de communautés dont les écrivains modernes ne sont pas toujours exclus. Rendu à son sens littéral, il peut être un espace de rencontre entre l'art et la foule, entre l'art et la vie.
\end{abstract}

Keywords : langue littéraire, lieu commun, modernité, valeur littéraire, communication littéraire

Il aura fallu à Jean Paulhan tout un cheminement, et plutôt encoléré, à travers l'histoire de l'idée de littérature, un parcours de Hugo à Bergson et aux surréalistes, pour conclure à l'injonction si joliment paradoxale qu'il propose d'afficher à l'entrée du jardin de Tarbes : « il est défendu d'entrer dans le jardin public sans fleurs à la main $»^{I}$. Substituée à la pancarte brandie par les «terroristes des lettres », visant au contraire à chasser ceux dont les mains seraient prises par quelque bouquet rhétorique cueilli ailleurs, le «nouvel écriteau » ouvre sur un récit que Paulhan ne raconte pas : bien que son panneau traduise " une mesure ingénieuse à tout prendre ", puisqu'il décourage les promeneurs de s'intéresser à d'autres fleurs que celles qu'ils tiennent déjà, il n'empêche qu'on rêve ensuite à ce qui pourrait « cependant » «arriv[er] peu après $»^{2}$. Clarifions tout à fait la fable allégorique : si Paulhan appelle de ses vœux une littérature qui n'aurait plus peur du langage, même courant, libre toutefois à d'autres que lui d'imaginer la forme ou l'histoire de leur réconciliation, et les promesses qu'elle pourrait contenir. C'est ce que nous allons faire ici.

Le lieu commun, que ce numéro envisage dans son rapport à la parole littéraire, fait l'objet d'une légende, laquelle en organise le détournement systématique, mais aussi d'une histoire, formulée justement dans la marge d'une autre plus glorieuse, celle de la littérature. La légende est connue : on dit que les écrivains abhorrent le lieu commun, que leurs plumes ne le to-

I Jean Paulhan, Les Fleurs de Tarbes ou la Terreur dans les lettres [1936], Paris, Gallimard, I94I, p. 165.

2 Ibid., p. 166. 
lèrent à la limite que pour s'en moquer. L'histoire est un peu plus complexe, elle remonte surtout plus loin : elle raconte comment la valeur du lieu commun s'est altérée au moment précis où émergeait le romantisme et rappelle que le topos avait été avant cela un instrument rhétorique dont personne ne songeait à contester l'utilité, le « siège des arguments » d'Aristote ${ }^{3}$, fournissant à l'orateur des « développements et preuves applicables à tous les sujets ", c'est-à-dire des formes et des idées lui permettant d'élaborer un discours compétent et solide. Plus tard, en France, le lieu commun devient un terme technique désignant deux dispositifs chers aux humanistes, $\mathrm{du} \mathrm{xVI}^{\mathrm{e}}$ jusqu'au XviII ${ }^{\mathrm{e}}$ siècles : un développement oratoire, d'abord, et ensuite, le mot étant alors mis au pluriel, un recueil constitué de citations et d'aphorismes frappants ${ }^{4}$. Mais le début de la modernité change tout : tandis que de leur côté les belles-lettres prennent le nom de littérature et adoptent ce faisant de nouvelles valeurs phares, celles d'authenticité, de singularité et surtout d'originalité - le mot est encore récent, il date du début du XVIII ${ }^{\mathrm{e}}$ siècle -, le lieu commun perd son prestige et vient s'appliquer aux idées et arguments rebattus. Le sens moderne du terme, qui est encore son sens courant aujourd'hui, s'impose si bien qu'il fait oublier que le lieu commun n'a pas toujours été haïssable. Un tel glissement sémantique révèle une mutation idéologique importante, qui n'est pas sans lien avec les turbulences sociales et politiques de l'ère post-révolutionnaire : c'est que la pensée de tous et la langue commune, devenues dangereuses, éveillent le soupçon des élites aristocratiques ou bourgeoises - auxquelles les écrivains appartiennent encore souvent. Le nouveau sens du lieu commun fournit à la toute nouvelle idée de littérature une contrepartie : l'originalité de l'une se démarque de la banalité de l'autre, la voix singulière de l'artiste romantique, qui lui permet d'exprimer son «moi » intime, s'oppose à une parole collective moins prestigieuse et moins profonde. L'invention du lieu commun moderne procure aussi à la littérature un nouveau rôle, celui de dénoncer les évidences et la médiocrité, parfois la bêtise, de cette voix collective qui n'appartient à personne : l'œuvre libérera son lecteur en lui permettant de s'élever au-dessus de la pensée commune. Rien d'étonnant à ce qu'un "témoin ${ }^{5}$ aussi avisé que le poète Stéphane Mallarmé, au mitan des années I880, ait été en

3 Sur les Topiques d'Aristote, voir le numéro 270 de la Revue internationale de philosophie, paru en 2014 et dirigé par Michel Meyer. Sur la notion d'endoxon chez Aristote, voir Peter I. Von Moos, «Introduction à une histoire de l'endoxon », in Lieux communs, topoï, stéréotypes, clichés, éd. Christian Plantin, Paris, Kimé, I993, pp. 3-I6.

4 Sur ces deux usages du terme, voir Francis Goyet, " Aux origines du sens actuel de "lieu commun" ", Cahiers de l'association internationale des études françaises, 49, I997, pp. 59-74. Sur les usages du lieu commun au XVII siècle, voir Bernard Beugnot, «Florilèges et Polyantheae. Diffusion et statut du lieu commun à l'époque classique ", Études françaises, I3:I-2, I977, pp. II9-I4I. 5 Stéphane Mallarmé, "Crise de vers ", in Euvres complètes, éd. Bertrand Marchal, 2 vol., Paris, Gallimard, « Bibliothèque de la Pléiade», t. II, p. 205. 
mesure de ressaisir dans une formule frappante la préoccupation des écrivains du siècle, depuis au moins deux générations : « un désir indéniable à l'époque est de séparer, comme en vue d'attributions différentes, le double état de la parole, brut ou immédiat ici, là essentiel $»^{6}$.

La valeur du lieu commun subit une variation spectaculaire, de l'adhésion enthousiaste des humanistes au mépris des romantiques et des modernes, dans le passage stupéfiant « d'une éloquence du lieu commun à une rhétorique du stéréotype $»^{7}$. Bien qu'un petit nombre de réhabilitations ait été tenté depuis Paulhan ${ }^{8}$, la critique littéraire s'est conformée à ce qu'elle a perçu comme une position dominante dans la modernité : le lieu commun y subit selon elle un traitement littéraire bien spécifique, un détournement au moins critique, souvent ironique. Habitués à valoriser l'originalité de l'œuvre littéraire, les modernes, s'ils consentent parfois à la présence de lieux communs, ne peuvent le faire à ses yeux qu'en passant par l'accusation, la subversion ou la parodie, sous peine d'entamer la valeur de leur propre écriture. Ainsi, la critique, encore soumise - et plus qu'elle ne veut bien l'admettre - à une idéologie romantique pourtant faiblissante depuis pas mal de temps, a eu beaucoup de peine jusqu'ici à envisager les rapports de la littérature et du langage courant. Son propos a été presque complètement homogène, jusqu'à devenir à son tour... commun.

En plus d'être à certains égards inquiétante, une telle uniformité attire le soupçon. À force de se mettre si bien d'accord, la critique n'a pas laissé de place au développement d'autres valeurs du lieu commun; en envisageant celui-ci, toujours, comme la cible dérisoire de l'éloquence moderne, elle ne s'est pas autorisée à y voir autre chose. Or, les dernières vingt années de travaux sur la littérature ont très clairement permis de réinscrire celle-ci dans un tissu discursif et de la réconcilier avec la rhétorique (réalisant ainsi le vœu de Paulhan). Le développement de la sociologie littéraire, des études de contextualisation et des travaux sur la discursivité littéraire ont fait tomber une barrière : on peut sans aucun doute, aujourd'hui, rouvrir la discussion et envisager la valeur du lieu commun avec plus de nuances, et plus de créativité, en tenant compte de plusieurs éléments importants que les travaux

6 Id., « Avant-dire au Traité du verbe de René Ghil », in EEuvres complètes, op. cit., t. II, p. 677.

7 Alain Vaillant, L'Histoire littéraire, Paris, Armand Colin, 20I0, p. 309.

8 Voir notamment Michael Riffaterre, «Fonctions du cliché dans la prose littéraire » [1964], in Essais de stylistique structurale, Paris, Flammarion, 1970, pp. I6I-I82 ; et Laurent Jenny, "Structure et fonctions du cliché. À propos des "Impressions d'Afrique" ", Poétique, 12, 1972, pp. 495517 .

9 Parmi quelques travaux récents, mais publiés de façon dispersée, on peut relever par exemple les articles de Marie-Chantal Killeen, «Duras et la littérature de quatre sous : un malentendu ?», French Studies, LXX, 4, 2016, pp. 550-564 ; Arnaud Bernadet, «Du poncif : littérature et malédiction ", in Deux siècles de malédiction littéraire. Transformations, médiations et transferts d'un mythe, éds. Pascal Brissette et Marie-Pier Luneau, Liège, Presses universitaires 
fondateurs de Ruth Amossy, dans les années 1980 et 1990, avaient déjà permis d'introduire. Même s'il y était question, comme de juste, d'observer le mode «critique » sur lequel la littérature se saisissait du lieu commun, pour " assur[er] [s]a différence et [s]a supériorité menacées " ${ }^{10}$, mais en admettant par ailleurs que « le cliché apparaît non seulement comme inévitable, mais aussi comme indispensable " ${ }^{\mathrm{II}}$, Amossy insistait sur les "stratégies discursives $»^{12}$ mises en place par la littérature face au lieu commun. Elle concevait ce dernier, à son tour, comme un objet discursif qu'il serait vain d'extraire de son contexte, c'est-à-dire des interactions verbales qui en déterminent la valeur.

Le présent numéro propose ainsi de changer la perspective : de ne plus se limiter à considérer les écrivains modernes comme les pourfendeurs ou les victimes du lieu commun, mais de voir qu'ils en sont aussi souvent les producteurs (qu'ils le veuillent ou non, d'ailleurs, ceux qui le veulent ayant évidemment des propos plus intéressants que les autres à ce sujet). Les articles rassemblés ici sont les résultats d'une expérience, ou d'un défi pour la pensée. On se proposait en effet, au départ, de prendre le contre-pied de la critique dominante, non sans quelque audace et au risque de forcer parfois le trait, mais avec une idée en tête, celle de penser (de contribuer à penser) le partage de la littérature, sur lequel des chercheurs aussi différents que Jacques Rancière ou Hélène Merlin-Kajman travaillent depuis plusieurs années. On a donc d'emblée postulé un refus de l'ironie, du côté de l'écrivain comme de celui du critique. Et l'on s'est posé la question, avec le plus grand sérieux : que fait la littérature au lieu commun ? Comment penser, aujourd'hui, un nouveau ou un autre rapport de la parole littéraire à la parole ordinaire?

La démarche n'est pas sans enjeu théorique, elle engage même une réflexion sur ce que peut être la valeur de la littérature, réflexion qu'il est toujours aussi difficile de mener de nos jours, bien après que Gérard Genette, Antoine Compagnon ou Jean-Marie Schaeffer, dans les années I990, en aient déclaré l'insolubilitét ${ }^{13}$. Quitte à ne pas lui apposer de dénouement,

de Liège, 20I4, pp. 99-I09 ; Suzanne Lafont, « Éloge du cliché chez Beckett dans En attendant Godot, La Dernière Bande, Oh les beaux jours », in Limit(e) Beckett, 2, 201I, pp. 37-52 ; Gaspard Turin, "Toussaint, Echenoz, Chevillard : le cliché comme forme d'engagement littéraire ", Versants, 52, 2006, pp. 73-96.

Io Ruth Amossy et Elisheva Rosen, Les Discours du cliché, Paris, Éditions SEDES, I982, p. 19.

II Ibid., p. I40.

I2 Ibid., p. 22. Voir aussi Ruth Amossy, Les Idées reçues. Sémiologie du stéréotype, Paris, Nathan, I99I ; Ruth Amossy et Anne Herschberg Pierrot, Stéréotypes et clichés. Langue, discours, société, Paris, Nathan, 1997 (l'ouvrage a fait l'objet de multiples rééditions dans les années 2000, la dernière en 2016 chez Armand Colin).

I3 Voir Gérard Genette, La Relation esthétique (L'EEuvre de l'art, t. II), Paris, Seuil, 1997, pp. 7II47 ; Antoine Compagnon, Le Démon de la théorie. Littérature et sens commun, Paris, Seuil, I998, pp. 267-304 ; Jean-Marie Schaeffer, Les Célibataires de l'art. Pour une esthétique sans mythes, Paris, 
la question mérite toutefois (et plus que jamais) d'être soulevée : il en va de cette ligne de partage qu'on dessine entre le littéraire et le non-littéraire, entre l'art et la « prose du monde $»^{14}$, qui bouge avec le temps et que les travaux de Rancière nous incitent à la fois à observer et à tenter de faire bouger. Il en va aussi de la « transitionnalité ${ }^{15}$ de la littérature, parce que la question implique de définir ses modes d'adresse, comme on le verra tout à la fin du numéro dans l'article de Jan Baetens.

On aimerait partir d'une énigme qui se pose encore aux spécialistes de poésie moderne : dans ses journaux intimes, Baudelaire profère trois déclarations sur le lieu commun, dont il est d'autant plus compliqué de savoir quoi faire qu'on connaît bien, pour le voir amplement à l'œuvre dans ses Salons, son dégoût du poncif (sur lequel l'article de Julien Zanetta reviendra plus loin). Les formules sont lapidaires, couchées sur le papier comme des aphorismes, sans lien explicite avec ce qui les précède ou les suit. Elles concernent en fait trois formes différentes de lieux communs, attribués à des collectivités distinctes : dans Hygiène, la sentence « rien de plus beau que le lieu commun ${ }^{16}$ fustige peut-être le " grand style » du littérateur, qui soigne son écriture jusque dans les marges de l'œuvre. Dans Fusées, Baudelaire affirme que "créer un poncif, c'est le génie ", avant de retourner l'axiome général en un projet pour soi-même : "Je dois créer un poncif ${ }^{17}$. S'il s'agit à ce moment-là de commenter le lieu commun artistique, fruit du travail (bon ou mauvais, ça n'est pas certain) du poète, une formule isolée du même cahier, au tout début de celui-ci, l'attribue quant à lui à la foule : «Profondeur immense de pensée dans les locutions vulgaires, trous creusés par des générations de fourmis $»^{18}$. Comme on le voit, ce n'est pas que les formules de Baudelaire soient équivoques ; on s'interroge plutôt sur l'éventuelle ironie avec laquelle il est possible qu'elles aient été proférées, ce qui est assez évident dans le premier cas, mais moins dans les deux suivants. Ça l'est supposément beaucoup lorsque le salonnier annonce à son correspondant, en I859, qu'il se résout au lieu commun par contagion d'avec « la banalité dans le peintre ", mais plus tant que ça lorsqu'il poursuit, sur un ton certes badin : « D'ailleurs, vous n'y perdrez rien ; car existe-t-il (je me plais à constater que vous êtes en cela de mon avis) quelque chose de plus charmant, de plus fertile et d'une nature plus positivement excitante que le lieu

Gallimard, I996, pp. 185-247.

I4 Jacques Rancière, Aisthesis. Scènes du régime esthétique de l’art, Paris, Galilée, 20II, p. II.

I5 Voir notamment Hélène Merlin-Kajman, «La Transitionnalité de la littérature : une dis/ continuité historique », in Fragments d'un discours théorique, éd. Emmanuel Bouju, Paris, Cécile Defaut, 2015, pp. 37I-400.

I6 Charles Baudelaire, Hygiène, in Euvres complètes, 2 vol., éd. Claude Pichois, Paris, Gallimard, « Bibliothèque de la Pléiade », t. I, p. 670.

I7 Id., Fusées, in Euvres complètes, op. cit., t. I, p. 662.

I8 Ibid., p. 650. 
commun ? " ${ }^{19}$. Dans l'essai sur Gustave Flaubert, encore, ce n'est pas l'ironie, toujours possible néanmoins, qui affleure le plus manifestement quand le poète reproche à Champfleury de « néglig[er] le lieu commun, le lieu de rencontre de la foule, le rendez-vous public de l'éloquence $»^{20}$.

Il reste par conséquent difficile de situer une fois pour toutes, et pour toutes les occasions, la valeur du lieu commun chez Baudelaire, comme chez Flaubert, qui, lorsqu'il imaginait d'écrire la préface de son Dictionnaire des idées reçues, promettait de l'« arrang[er] de telle manière que le lecteur ne sache pas si on se fout de lui, oui ou non $»^{21}$. Les hésitations de la critique sont donc bien concevables, et même plutôt saines : suspendre l'enquête sur la valeur du lieu commun chez les écrivains modernes, c'est en effet prendre le risque de céder à l'idéologie, c'est refuser de voir, par exemple, comme les lecteurs ont eu tendance à le faire à partir des années I920, que Baudelaire a beau avoir été un maître de l'innovation en théorie, sa pratique poétique montre qu'il a pourtant recouru fréquemment au lieu commun ${ }^{22}$, sachant sans doute qu'en quantité raisonnable, il est ce dont la perception a besoin pour reconnaître la présence d'une œuvre d'art, comme l'ont montré les travaux d'Ernst Gombrich ${ }^{23}$.

Reste ainsi que chez Baudelaire, et chez Flaubert aussi - « le lieu commun n'est manié que par les imbéciles et les très grands ${ }^{24}$, écrit-il à Louise Colet en I853 -, c'est-à-dire chez le poète et le prosateur les plus significatifs du début de la modernité, avec ou sans ironie (on ne le sait pas), le lieu commun apparaît comme une condition, et même la condition ultime, du génie : le pouvoir qui lui est propre, c'est celui-ci que doit conquérir l'écrivain - l'idée est encore à retrouver chez Paulhan, qui rappelle, avec le plus grand sérieux cette fois-ci, que « pour banal que soit un lieu commun, il peut toujours avoir été inventé par qui le prononce : il s'accompagne même, dans ce cas, d'un vif sentiment de nouveauté ${ }^{25}$. Au sein même des œuvres les plus apparemment acquises à la dénonciation du poncif demeurent des zones d'incertitude, des zones possiblement contestataires, qui pourraient bien esquisser, ou seulement contempler, du cœur de la modernité le ren-

I9 Id., Salon de I859, in Euvres complètes, op. cit., t. II, pp. 608-609.

20 Id., Madame Bovary par Gustave Flaubert, in Euvres complètes, op. cit., t. II, p. 79.

2I Gustave Flaubert, lettre à Louis Bouilhet, 4 septembre I850, in Correspondance, 5 vol., éd. Jean Bruneau, Paris, Gallimard, « Bibliothèque de la Pléiade », I973-2007, t. I, p. 679.

22 À ce propos, voir Jonathan Culler, «Poésie et cliché chez Baudelaire », in Le Cliché, éd. Gilles Mathis, Toulouse, Presses universitaires du Mirail, 1998, pp. 205-2I8 ; et Didier Alexandre, "Chants du cygne. Le cliché et sa prévisibilité en poésie », in Lieux communs, topoï, stéréotypes, clichés, op. cit., pp. 46-59.

23 Voir Ernst Gombrich, L'Art et l'Illusion. Psychologie de la représentation picturale, trad. Guy Durand, Paris, Gallimard, I97I.

24 Flaubert, lettre à Louise Colet, 2 juillet I853, in Correspondance, op. cit., t. II, p. 372.

25 Paulhan, Les Fleurs de Tarbes, op. cit., pp. 85-86. 
versement de celle-ci.

Il existe aussi des voix dissidentes au sein du romantisme, celle de Charles Nodier par exemple, que l'article de Nicolas Wittwer met à l'honneur. Bien que ce soit certainement aux romantiques, et en particulier aux romantiques allemands, qu'on doit le clivage moderne entre langue littéraire et langue courante, il n'empêche que certains d'entre eux célèbrent avec conviction les vertus du commun. Il y a du romantisme en abondance chez Nodier, même s'il n'adhère pas à la doctrine de l'originalité et que sa réflexion sur le style ne passe pas par un rejet du commun. Son romantisme se manifeste plutôt dans des spéculations fantasmagoriques sur les origines de la langue, dans un imaginaire qui attribue aux expressions banales une valeur poétique, du fait de leur plus grande proximité avec une parole primitive, idéale, et toujours à retrouver ou à refonder. Mais le fantasme, qui réapparaîtra bizarrement chez Paulhan, aussi tardivement que dans les années 1930, excède la rêverie et informe à plusieurs égards le travail d'écrivain de Nodier, à travers la défense d'un " style singulièrement commun ", dans un discours critique qui s'oppose par exemple au néologisme, prônant plutôt des valeurs telles que la simplicité et la naïveté, et jusque dans le choix d'un genre privilégié, celui du conte. "Événement du langage », " signe d'un triomphe $»^{26}$, le lieu commun que Nodier considère comme témoin ou reste d'une littérarité primordiale, mais perdue, Paulhan en soulignera de la même façon les pouvoirs, son propos prenant alors la même tonalité mythologique.

Quand elle en admet la présence, que fait la littérature au lieu commun ? Le numéro, à travers les quatre articles qui suivent, développe une hypothèse : il y apparaît que la littérature permet de mobiliser, et même de revivifier les lieux communs, les restituant aux investissements pluriels de ses lecteurs et invitant ceux-ci à renégocier leur contenu. L'article de Maria Gal, ainsi, après avoir rappelé à partir d'Aristote que le lieu commun est un "mode d'expression didactique », se penche sur l'œuvre de Balzac et montre que si didactisme il y a, ce n'est certainement pas sur un mode autoritaire mais à travers la participation active des lecteurs, auxquels le texte fournit quantité de contenus instables, ambivalents. La réception des clichés, dont l'écriture de Balzac foisonne parfois, consiste non pas en leur entérinement mais en leur relativisation : le texte force ses lecteurs à varier les perspectives, qui font varier le sens, à moduler de plusieurs manières un même axiome, il « exploite [le lieu commun] jusqu'au bout de ses possibilités ». C'est le cas en particulier des dualismes donc Balzac est friand et que la critique a souvent commentés : Gal montre qu'ils sont mobiles, repris et travaillés, "dramatisés » jusqu'à ce que les lecteurs, auxquels un tel dispo-

26 Ibid., pp. 163-I64. 
sitif s'adresse, comprennent que c'est à eux qu'il revient de décider de leur sens, ou mieux, de ne pas en décider du tout.

La littérature, qui ne touche pas à la forme stable du lieu commun, affecte pourtant radicalement sa stabilité idéologique. Sa récursivité n'y est de fait plus que superficielle : on a déjà vu que la valeur du lieu commun est fluctuante, et il semble que grâce au traitement que lui fait subir le discours littéraire, son sens peut devenir précaire à son tour, et donc récupérable par chacun. À la limite, le lieu commun répond à un fantasme qu'il n'est pas rare de rencontrer chez les modernes, celui d'une littérature purifiée de tout contenu, qui ne tiendrait que par sa forme. Il pourrait dans ce cas jouer le rôle, pour la parole littéraire, d'un modèle absolu - statut que Ponge n'hésite pas à attribuer parfois au proverbe, dans les termes les plus explicites. Mon article, à partir du bref essai que celui-ci consacre à Mallarmé dans les années I920, met à l'épreuve et confirme les déclarations audacieuses qui s'y donnent à lire, lesquelles font de l'auteur du Coup de dés, déjà considéré comme l'un des pères de la modernité et des avant-gardes, un producteur de lieux communs, déclinés ici en formules, maximes et proverbes. Avec Ponge et Mallarmé, on s'intéresse à ce qui se produit lorsqu'une forme devient formule, surtout s'il s'agit de formes et de formules littéraires, passibles de s'opposer aux discours concurrents de la science, de la politique ou du droit. Pour que l'opération réussisse, il faut impérativement que la forme soit vidée de son contenu, afin que celui-ci puisse être approprié par le lecteur, comme chez Balzac : la littérature travaille ainsi soit à invalider le sens des formules en utilisant celles-ci à contre-emploi, soit à le rendre superflu en s'assurant qu'il n'est plus que tautologique (ou, chez Mallarmé en particulier, réflexif). La vision de Ponge, ouvertement pragmatique, incite le lecteur à déceler chez Mallarmé, à son tour, un intérêt pour les usages de la poésie, le souci de garantir à ses formules la plus grande efficacité possible.

Tandis que l'article de Nils Couturier sur Laforgue montrera lui aussi, plus tard, qu'une utilisation poétique des clichés permet de réactiver leur sens, souvent sur un mode ludique mais non sans prendre au sérieux leur valeur de vérité, due à leur universalité et à leur origine populaire, les réflexions que mènent Daniele Carluccio sur Barthes et Natacha Allet sur La Demande de Michèle Desbordes permettent d'illustrer, d'un point de vue cette fois pratique, la façon dont l'art et la littérature s'emparent du contenu du lieu commun pour le revitaliser (et le terme peut être compris quasiment au littéral). À partir d'une autre perspective, on n'assiste plus à la manière dont le lieu commun se vide, mais à celle dont il se remplit à nouveau.

S'intéressant à Barthes, dont on connaît les déclarations pour le moins brutales à l'égard du stéréotype - « ce monstre » qui « dort » « en chaque 
signe $»^{27}-$, l'article de Carluccio retrace la quête tragique de l'auteur des Mythologies, à la recherche d'un lieu commun qui ne serait pas sentence répétitive, mortifère, mais remémoration ou même résurrection, d'un mythe derrière lequel il y aurait " quelqu'un ». Et la rédemption du stéréotype a bien lieu, ne serait-ce que fugacement, en deux instants auxquels Carluccio prête attention : dans la lecture de textes de Michelet sur Napoléon d'abord, puis surtout dans une réflexion sur le catch. Le spectacle des catcheurs que commente Barthes est en effet celui de corps typifiés qui n'ont pourtant rien perdu de leur humanité. Ce n'est certainement pas dans la langue, mais plutôt malgré elle que le stéréotype se trouve brièvement racheté : c'est dans l'art, dans la mise en spectacle de l'homme par l'homme, que Barthes considère comme une véritable tragédie (mais qui n'est pourtant plus très loin de l'outrance de la commedia dellarte), ou encore dans le choix de l'artefact, aux dépens de l'effet de réel que le cliché photographique produit par exemple, souvent de façon perverse. Dans le «tableau vivant » que donnent les catcheurs, sous les yeux de Barthes, le type se substantifie et reprend corps.

Le geste du catcheur, dit-il encore en empruntant la formule à Baudelaire, possède une "vérité emphatique »; dans d'autres cas aussi, ceux qu'observeront par exemple Margot Favard ou Isabelle Pitteloud, c'est à travers l'excès que le lieu commun, poussé vers un certain paroxysme, est mobilisé, repris et revisité. Mais pas chez Michèle Desbordes, qui, adoptant dans La Demande le genre des vies, récrit la légende - devenue commune - du plus emblématique de tous les peintres, Léonard de Vinci, en la déplaçant vers un lieu marqué par le commun, qu'elle traite avec douceur. De ce roman qui décrit sans ironie la rencontre et l'intimité croissante d'un grand artiste et de sa servante, l'article de Natacha Allet montre qu'il relève d'un engagement, en quelque sorte politique : il restitue le geste précis qu'inventait Vinci pour La Joconde, celui de mains croisées sur le tissu des jupes, devenu l'un des motifs les plus communs de l'histoire de l'art occidental, aux femmes qui, sans le savoir, l'inventaient avant lui dans la vie de tous les jours. Les «frontières de l'art et de la vie » sont franchies et refranchies dans la fiction, qui se donne tous les droits, même celui de ne pas tenir compte de la véritable chronologie, laquelle situe la réalisation du tableau avant la rencontre avec la servante, c'est-à-dire avant que Léonard se trouve fasciné par ses mains croisées et recroisées. Il n'empêche que le motif est rendu à son lieu d'origine, qu'il acquiert une deuxième fois le statut de lieu commun : ces mains croisées, qui ne sont peut-être au départ, pour le lecteur, rien d'autre qu'un motif artistique, les personnages du roman les investissent de sens qui les touchent, les définissent, les rattachent à un passé qu'ils confrontent

27 Roland Barthes, «Leçon inaugurale de la chaire de sémiologie littéraire au Collège de France prononcée le 7 janvier 1977 », in Euvres complètes, 5 vol., éd. Éric Marty, Paris, Seuil, 2002 , t. V, p. 432. 
à nouveau. La fiction donne à voir le remplissage du lieu commun, la reprise de sa forme, dans laquelle les personnages projettent des contenus intimes, développant à l'entour de celui-ci le lien qui les unit peu à peu et réduisant par là l'écart, instauré par la pensée moderne, entre l'art et la vie. Si le geste de Desbordes est politique, c'est qu'il consiste à rendre un motif commun à ceux qui n'ont pas eu le génie de le produire.

Quelle que soit la valeur qu'on lui attribue et les usages dans lesquels on le prend, il est certain que le lieu commun permet de fabriquer du collectif, de plusieurs manières. Le livre d'Anne Cauquelin sur L'Art du lieu commun (1999) insiste dès ses premières pages sur ce sens communautaire, qui met en jeu la valeur de la parole banale : « le commun, écrit-elle, peut être considéré aussi comme ce qui est en partage, à la source du sentiment communautaire, bienvenu pour un esprit démocratique $»^{28}$. Ainsi la locution « lieu commun » peut-elle être remobilisée, « en interrogeant ce qu'y signifie le mot commun $»^{29}$, comme proposent de le faire Lise Forment, Tiphaine Pocquet et Léo Stambul dans un ouvrage collectif consacré récemment à la Politique des lieux communs, avec l'ambition d'en faire resurgir la valeur humaniste. Or, plusieurs sortes de lieux communs - à différentes échelles dont Baudelaire était bien conscient, comme on l'a vu plus haut - fabriquent plusieurs formes de communauté, qui peuvent être restreintes (c'est le cas des communautés artistiques ou littéraires) ou très vastes (celle que contemplait Balzac liait l'auteur à tous les lecteurs possibles, celle qu'envisageait Mallarmé le liait même au-delà à tous ceux qui ne le lisent pas). Il existe ainsi des lieux communs savants, ceux que fustigeait Nodier, des lieux communs prestigieux, censés rassembler les élites. Et la question se pose par conséquent de savoir quels types de communautés la littérature est à même de fonder, sachant que les regroupements de tous ordres ont souvent pour conséquences des effets d'exclusion : s'agit-il de construire la communauté des (bons) artistes et des (bons) interprètes, contre les mauvais, les naïfs, les indifférents ou les idiots? Mais la logique des happy few qu'inventait le romantisme, en faisant des actes de lire et d'écrire des gestes individuels et centrés sur le " moi », n'est pas la seule possible, comme on a déjà commencé à le voir.

Observer les rapports du lieu commun et de la parole littéraire permet d'éclairer la socialité dont cette dernière est porteuse, l'un comme l'autre étant moins des objets (dont il serait possible de désigner les caractéristiques et la structure) que des jugements de valeur, relatifs et donc variables. Ils relèvent de l'idéologique, comme on l'a vu : s'il n'y a ni lieu commun, ni parole littéraire en soi, mais seulement pour quelqu'un qui en mesure la valeur, ce que montraient déjà les travaux d'Amossy mentionnés plus haut,

28 Anne Cauquelin, L'Art du lieu commun. Du bon usage de la doxa, Paris, Seuil, 1999, p. 9.

29 Lise Forment, Tiphaine Pocquet et Léo Stambul (dirs.), Politique des lieux communs, Rennes, Presses universitaires de Rennes, 20I6, p. II. 
force est d'admettre que leur commentaire s'adosse en premier lieu sur un repérage forcément délicat parce que jamais complètement objectif, pris dans des enjeux de hiérarchisation et de rivalité. On est peut-être toujours le lieu commun de quelqu'un d'autre, se dit-on au terme de la lecture de l'article de Julien Zanetta, qui porte sur la critique d'art de Baudelaire et de son contemporain Théophile Silvestre, ainsi que sur un roman des frères Goncourt, Manette Salomon. Pour les deux salonniers, la dénonciation du poncif, qui se fait en les termes les plus spirituels possibles, devient le lieu commun autour duquel gravite la communauté des critiques d'art. Dans une configuration sociale bien précise et marquée par des enjeux de pouvoir, où le ridicule guette, le poncif, brandi comme une insulte, sert surtout à montrer que l'un (en l'occurrence le critique) est plus malin que l'autre (le peintre, transformé dès lors soit en rapin, soit en académicien). Dans leurs écrits sur la peinture, Baudelaire et Silvestre emploient systématiquement le mot de "poncif " plutôt que celui de " lieu commun ", pour dénoncer un geste mécanique, une technique sans âme, une forme vide. Dans la fiction des Goncourt, il devient pourtant un moyen de réussir : avec un formidable cynisme, les romanciers reprennent le poncif, objet de la colère bien réelle des salonniers, de façon plus légère et en le théâtralisant. Ils en font l'un des numéros qui se donnent sur la scène de l'art, le jeu de sa dénonciation en étant peut-être un autre.

Il est clair que ni l'art, ni la littérature, même modernes, ne sont à l'abri du lieu commun, et qu'ils en produisent même leurs lots. En se formulant cette évidence, on se donne les moyens de toucher à leurs mythes, et en particulier à celui de l'artiste incompris, maudit ou obscur, comme le montrent les articles de Nils Couturier sur Laforgue et de Margot Favard sur Mallarmé. L'habitude qu'on a prise d'associer le succès commercial des œuvres à leur médiocrité, cautionnée scientifiquement par les travaux de Bourdieu, ne résiste pourtant pas à la distance temporelle : quand les lieux communs se déplacent et que leurs contenus varient, se redisposent aussi les effets de prestige et les positions d'autorité. Le lieu commun d'une communauté et d'une époque données n'est pas celui d'un autre temps et d'un autre groupe: aujourd'hui, Mallarmé est beaucoup plus lu, et beaucoup plus commun, qu'un Victorien Sardou par exemple, dont il commentait les vaudevilles à succès, à la fin du XIX ${ }^{\mathrm{e}}$ siècle, dans des chroniques alors pratiquement illisibles.

La réflexion de Couturier cherche à faire sens d'un paradoxe qu'il rencontre chez Laforgue : le personnage de Pierrot, du solitaire par excellence, s'y trouve mis au pluriel. Après avoir rappelé tout ce que l'étiquette de poète maudit, déjà commune au début de la modernité, inspire de méfiance à l'auteur des Complaintes, et souligné l'usage que ce dernier fait du motif commun qu'est la lune, Couturier met le doigt sur le problème : les poètes-Pierrots ont 
beau être des symboles de l'isolement poétique, leur surgissement dans la communauté littéraire de l'époque permet d'incarner les valeurs et l'idéal dont elle se soutient. Quand les personnages marginaux se multiplient, ils fondent, toujours à distance, des groupes paradoxaux, institués autour d'un imaginaire auquel Laforgue demande aussi à son lecteur d'adhérer. Une logique similaire guide les stratégies qui permettent à Mallarmé de s'imposer comme une figure à la fois commune et singulière, dans le paysage littéraire de la deuxième moitié du siècle, «bizarre » à force de grossir les traits d'un personnage pourtant conforme aux valeurs poétiques du moment. L’article de Favard décrit la façon dont « Mallarmé l'obscur » fabrique son obscurité, dont il emprunte l'idée notamment à Victor Hugo, mais qui finit par le « sortir du commun ». Malgré l'étrange cécité des poètes, parfois, devant leurs propres lieux communs, le cas de Mallarmé permet de montrer pourquoi il n'est pas vain qu'ils adoptent eux aussi les habitudes de pensée les plus courantes, afin d'obtenir la reconnaissance de leurs pairs et de fonder ainsi leur propre valeur littéraire. Favard montre que, pour Mallarmé, c'est par l'obscurité qu'il faut passer afin d'arriver à la gloire. Mais l'obscurité dont il s'agit fait l'objet d'un investissement rhétorique, et pas affectif : le poète fabrique une figure du poète obscur sans être pourtant désespéré. En travaillant avec subtilité les lieux communs de leur institution, il arrive en effet que les écrivains fassent la double preuve de leur capacité à comprendre les enjeux sociaux de celle-ci, et à agir librement et avec créativité dans les cadres qu'elle impose.

Tout comme Mallarmé, Flaubert est devenu, à force d'être lu, un lieu commun de la culture française : l'article d'Isabelle Pitteloud interroge la manière dont une écrivaine, Marie-Hélène Lafon, peut aujourd'hui justifier qu'elle y fasse recours, et sans céder à la parodie, dans le roman très visiblement intertextuel qu'est Joseph, réécriture d'Un cœur simple. Il apparaît que Lafon ne manque pas d'arguments pour le revendiquer : le lieu commun littéraire qu'est Flaubert lui sert de forme discursive, dans laquelle faire tenir la parole informe d'un ouvrier agricole, d'abord. Il lui permet de résoudre un problème d'écriture, celui de faire un livre sur un personnage qui ne s'exprime que par lieux communs, sans renoncer à sa banalité mais sans mettre en péril la valeur de sa mise en récit; il lui permet en d'autres termes de faire surgir la parole commune dans le texte littéraire, en préservant la valeur de l'une comme de l'autre. L'article de Pitteloud montre aussi que l'intertexte, ce motif commun de la grande et de la vaste cultures - on rappellera que Flaubert est lu dans les écoles, enseigné par les « profs » auxquels Lafon se trouve liée plus ou moins malgré elle -, permet de déplacer les relations de pouvoir entre dominant et dominé, dans le récit d'abord, et même pour l'auteur elle-même, l'espace d'un instant « révolutionnaire " pendant lequel le lieu commun change de mains. Dans le geste de Lafon, lui 
aussi politique d'une certaine manière, il s'agit de retirer au génie le pouvoir de créer à lui seul tous les poncifs, en montrant qu'un même lieu commun peut fonder plusieurs sortes de communautés.

Si l'histoire, littéraire, des valeurs du lieu commun témoigne de l'évolution des rapports entre l'écrivain et l'homme ordinaire, elle permet enfin de réfléchir à ceux qui lient l'auteur et ses lecteurs, rapports dont la théorie a elle aussi bien changé au cours du temps : de la connivence à la rivalité et retour, l'adresse au lecteur a pris de multiples formes, que l'étude du lieu commun, qualifié par Amossy d'« effet de lecture $»^{30}$, permet d'appréhender un peu de biais. Il semble que le lieu commun fasse apparaître la fragilité de la position d'auteur, qui n'est en effet que temporaire : n'en déplaise à Baudelaire, le génie qui « crée un poncif » s'efface aussitôt après, pour le léguer aux lecteurs et le laisser vivre sa propre vie, comme le racontait Ponge dans l'une de ses fables allégoriques. Dès qu'elle devient commune, la locution s'impersonnalise pour mieux se rendre disponible au plus grand nombre.

Le poète ne fait que "passer », semble-t-il, comme dans le titre du roman de Ramuz, Passage du poète, dont Valérie Bucheli commente plusieurs extraits. Il « passe» parmi les hommes pour leur montrer que leur usage de la langue, bien qu'il n'ait sans doute pas le prestige d'un usage littéraire, n'est pas pour autant dénué de valeur - de valeur esthétique comme de valeur de vérité, ce à quoi Mallarmé n'aurait peut-être pas trouvé à redire, lui qui se demandait « à quoi bon la merveille de transposer un fait de nature en sa presque disparition vibratoire, [...] si ce n'est pour qu'en émane [...] la notion pure ${ }^{31}$. Dans l'article de Bucheli, c'est encore par l'intertextualité que s'accomplit la revalorisation du lieu commun : le roman de Ramuz rejoue délibérément la scène des Comices racontée par Flaubert dans Madame Bovary, mais en y faisant varier les effets de regroupement, en redistribuant le pouvoir et les valeurs, selon un engagement que Bucheli qualifie d'humaniste. Le sens même du mot "médiocrité » est peut-être à revoir, à neutraliser : la moyenne ne se démarque plus par sa bêtise, chez Ramuz, mais elle est à l'origine de rapprochements humains qui remettent en jeu les étagements hiérarchiques. Dans l'œuvre de l'écrivain suisse, le lecteur ne lit plus nécessairement la langue d'un autre, cette « langue étrangère » que Proust associait à tous les « beaux livres $»^{32}$, mais sa langue à lui, dont il voit bien qu'elle n'est pas dénuée de beauté. Le style du poète n'est plus l'expression d'un seul individu mais il s'imprègne d'une voix collective, celle d'une communauté habituellement dévalorisée, dont la littérature montre la dignité.

Le numéro se termine sur un article signé par Jan Baetens, critique

30 Amossy et Rosen, Les Discours du cliché, op. cit., p. 9.

3I Mallarmé, "Avant-dire au Traité du verbe de René Ghil », art. cit., p. 677.

32 Marcel Proust, Contre Sainte-Beuve précédé de Pastiches et mélanges et suivi de Essais et articles, éd. Pierre Clarac, Paris, Gallimard, « Bibliothèque de la Pléiade », 1971, p. 299. 
littéraire mais aussi poète, dont les recueils, Pour une poésie du dimanche, Autres nuages, Le Problème du Sud, Ce monde ou La Lecture, pour ne citer que quelques-uns des plus récents d'entre eux, méritent d'être repris ou découverts. C'est donc en poète que Baetens écrit ici : le lieu commun, admet-il, pose à l'écrivain une question fondamentale, celle de la manière dont il s'adresse à ses lecteurs. C'est grâce à une petite quantité (raisonnable) de lieux communs, comme on l'a vu, qu'un artiste se fait reconnaître comme tel d'abord, puis comprendre par son public ensuite. Pour Baetens, à une époque où les références culturelles de l'un ne sont plus nécessairement partagées par l'autre (qui n'est d'ailleurs en rien homogène), se pose le problème de savoir comment communiquer par allusions, étant entendu que la littérature éprouve aujourd'hui très sérieusement le besoin de produire $\mathrm{du}$ commun. Comment faire, du point de vue de l'auteur, pour s'assurer que son geste d'écriture rencontre un geste de lecture qui lui convienne, mais sans contrôler abusivement l'activité herméneutique de son public ? Baetens, à partir d'un recueil d'Emmanuel Hocquard puis de l'un des siens, expose une solution pratique, consistant à fabriquer un dispositif qui expliciterait aux alentours du texte, dans des péritextes eux-mêmes textualisés, les allusions qui en contraignent la réception, de manière à ce qu'elles ne la limitent plus. Baetens insiste sur la nécessité de textualiser l'allusion, et l'on voit bien pourquoi : à travers un tel procédé, dont les variations sont multiples, l'attention se déplace, du contenu du texte vers les modalités de sa transmission. Le poème ne se referme pas sur lui-même, mais il se retourne sur ses moyens d'adresse, sur sa transitivité, internalisée. Dans le dernier exemple que donne Baetens, celui d'un poème sur Venise, qui ne parle pas de Venise mais d'un artiste y ayant séjourné (suffisamment peu connu pour que nombre d'allusions à son sujet se perdent, n'était le dispositif qui sous les yeux du lecteur les récupère), on se prend au jeu du déchiffrage, mais en étant amené, par le texte, à observer la manière dont le sens nous parvient, c'est-à-dire la manière dont le poème se donne en partage ${ }^{33}$.

33 La réflexion dont ce numéro procède a été amorcée lors d'un colloque organisé à l'Université de Genève les 20 et 2I octobre 20I6, avec le soutien du Fonds national suisse pour la recherche scientifique et la participation de Pascal Durand, Philippe Geinoz et Martin Rueff, auxquels j'adresse mes remerciements. 